\title{
ON A CERTAIN CORRESPONDENCE BETWEEN SURFACES IN HYPERSPACE*
}

\author{
BY \\ V. G. GROVE

\section{INTRODUCTION}

Consider a surface $S$ and a point $x$ on $S$. Let the parametric vector equation of $S$ be

$$
x=x(u, v) .
$$

The ambient space of the osculating planes at the point $x$ to all of the curves through $x$ is a certain space $S(2,0)$ called the two-osculating space of $S$ at $x$. This space is determined by the six points

$$
x, x_{u}, x_{v}, x_{u u}, x_{u v}, x_{v v} .
$$

It is the purpose of this paper to find all surfaces $\bar{S}$ in one-to-one point correspondence with $S$, such that the two-osculating space $\bar{S}(2,0)$ of $\bar{S}$ coincides with the two-osculating space $S(2,0)$ of $S$ at corresponding points. We shall find that the surface $S$ is not arbitrary, but that the functions $x$ satisfy certain third-order partial differential equations studied by Lane $\dagger$ and by Bompiani. $\ddagger$ A similar statement holds for the surface $\bar{S}$.

Let the surfaces $S$ and $\bar{S}$ be in one-to-one point correspondence so that the corresponding points have the same curvilinear coordinates.

In order that $\bar{S}(2,0)$ at $\bar{x}$ coincide with $S(2,0)$ at $x$, it is necessary and sufficient that the functions

$$
\bar{x}, \bar{x}_{u}, \bar{x}_{v}, \bar{x}_{u u}, \bar{x}_{u v}, \bar{x}_{v v}
$$

be expressible as linear, homogeneous functions of the functions (2). The parametric vector equation of $\bar{S}$ will therefore be of the form

$$
\bar{x}=\bar{x}(u, v)=A x_{u u}+B x_{u v}+C x_{v v}+\alpha x_{u}+\beta x_{v}+\gamma x .
$$

We shall call the case in which $S(2,0)$ is a space of five dimensions and in which the coefficients $A, B, C$ of (4) satisfy the inequality

\footnotetext{
* Presented to the Society, April 7, 1934; received by the editors February 20, 1934.

$\dagger$ E. P. Lane, Integral surfaces of pairs of partial differential equations of the third order, these Transactions, vol. 32 (1930), pp. 782-793. Hereafter referred to as Lane, Surfaces.

¥ E. Bompiani, Determinazione delle superficie integrali d'un sistema di equazioni a derivate parziali lineari ed omogenee, Rendiconti del Reale Istituto Lombardo di Scienze e Lettere, vol. 52 (1919), pp. 820-830. Hereafter referred to as Bompiani, Surfaces.
} 


$$
B^{2}-4 A C \neq 0
$$

the non-parabolic case, and the case in which $S(2,0)$ is a space of five dimensions and in which

$$
B^{2}-4 A C=0
$$

the parabolic case. By proper choice of $\phi, \psi$, and $\lambda$ in the transformation

$$
\bar{u}=\phi(u, v), \quad \bar{v}=\psi(u, v), \quad \bar{x}=\lambda \bar{x}^{\prime},
$$

in the non-parabolic case, we may write (4) in the form

$$
\bar{x}=x_{u v}+\alpha x_{u}+\beta x_{v}+\gamma x ;
$$

and in the parabolic case in the form

$$
\bar{x}=x_{u u}+\alpha x_{u}+\beta x_{v}+\gamma x .
$$

We shall denote by $S(3,0)$ the ambient space of the three-dimensional spaces osculating all of the curves on $S$ through $x$. The space $S(3,0)$ is determined by the six points (2) and the points

$$
x_{u u u}, x_{u u v}, x_{u v v}, x_{v v v} \text {. }
$$

\section{The NON-PARABOLIC CÀSE}

If we differentiate $\bar{x}$ defined by (7) with respect to $u$ and $v$ we obtain the following expressions:

$$
\begin{aligned}
& \bar{x}_{u}=x_{u u v}+\alpha x_{u u}+\beta x_{u v}+\left(\alpha_{u}+\gamma\right) x_{u}+\beta_{u} x_{v}+\gamma_{u} x, \\
& \bar{x}_{v}=x_{u v v}+\alpha x_{u v}+\beta x_{v v}+\alpha_{v} x_{u}+\left(\beta_{v}+\gamma\right) x_{v}+\gamma_{v} x .
\end{aligned}
$$

The points $\bar{x}_{u}, \bar{x}_{v}$ are in the space $S(2,0)$ if, and only if, the functions $x$ defining the surface $S$ satisfy a system of differential equations of the form

$$
\begin{aligned}
& x_{u u v}=a x_{u u}+h x_{u v}+b x_{v v}+l x_{u}+m x_{v}+d x, \\
& x_{u v v}=a^{\prime} x_{u u}+h^{\prime} x_{u v}+b^{\top} x_{v v}+l^{\prime} x_{u}+m^{\prime} x_{v}+d^{\prime} x .
\end{aligned}
$$

It follows therefore that in the non-parabolic case $S(3,0)$ is of dimensions no higher than seven.

Subcase a. Suppose that $S(3,0)$ is a space of seven dimensions. It follows that the functions $x$ satisfy the equations (11) and no other third-order differential equations. Under these conditions some of the integrability conditions* of system (11) are

$$
a^{\prime}=b=0, a h^{\prime}+l^{\prime}-a^{2}-a_{v}=0, b^{\prime} h+m-b^{\prime 2}-b_{u}^{\prime}=0 .
$$

Equations (10) may be written in the form

* Bompiani, Surfaces, p. 632. 


$$
\begin{aligned}
& \bar{x}_{u}=(a+\alpha) x_{u u}+(h+\beta) x_{u v}+\left(l+\alpha_{u}+\gamma\right) x_{u}+\left(m+\beta_{u}\right) x_{v}+\left(d+\gamma_{u}\right) x, \\
& \bar{x}_{v}=\left(h^{\prime}+\alpha\right) x_{u v}+\left(b^{\prime}+\beta\right) x_{v v}+\left(l^{\prime}+\alpha_{v}\right) x_{u}+\left(m^{\prime}+\beta_{v}+\gamma\right) x_{v}+\left(d^{\prime}+\gamma_{v}\right) x .
\end{aligned}
$$

From (12) we see that the points $\bar{x}_{u u}, \bar{x}_{u v}, \bar{x}_{v v}$ lie in $S(2,0)$ if, and only if,

$$
\alpha+a=0, \beta+b^{\prime}=0 .
$$

Therefore the point $\bar{x}$ defined by the expression

$$
\bar{x}=x_{u v}-a x_{u}-b^{\prime} x_{v}+\gamma x
$$

generates a surface $\bar{S}$ whose two-osculating space $\bar{S}(2,0)$ at $\bar{x}$ coincides with the two-osculating space $S(2,0)$ at $x$.

From (12) and (14) we find that the expressions for $\bar{x}_{u}$ and $\bar{x}_{v}$ may be written in the form

$$
\begin{aligned}
& \bar{x}_{u}=\left[a\left(h-b^{\prime}\right)+l-a_{u}+\gamma\right] x_{u}+\left[d+\gamma_{u}+\gamma\left(h-b^{\prime}\right)\right] x+\left(h-b^{\prime}\right) \bar{x}, \\
& \bar{x}_{v}=\left[b^{\prime}\left(h^{\prime}-a\right)+m^{\prime}-b_{v}^{\prime}+\gamma\right] x_{v}+\left[d^{\prime}+\gamma_{v}+\gamma\left(h^{\prime}-a\right)\right] x+\left(h^{\prime}-a\right) \bar{x} .
\end{aligned}
$$

Therefore the lines $g$ joining corresponding points $x$ and $\bar{x}$ of $S$ and $\bar{S}$ form $a$ congruence $G$, and the surfaces $S$ and $\bar{S}$ sustain $C$ nets* in relation $C$; the developables of $G$ intersect $S$ and $\bar{S}$ in" these $C$ nets. Conversely if two nets are in relation $C$ their sustaining surfaces have coincident two-osculating spaces at corresponding points.

Subcase b. Suppose that $S(3,0)$ is of six dimensions. By proper choice of the notation, the functions $x$ satisfy a system of differential equations of the form

$$
\begin{aligned}
& x_{u u v}=a x_{u u}+h x_{u v}+b x_{v v}+l x_{u}+m x_{v}+d x, \\
& x_{u v v}=a^{\prime} x_{u u}+h^{\prime} x_{u v}+b^{\prime} x_{v v}+l^{\prime} x_{u}+m^{\prime} x_{v}+d^{\prime} x \\
& x_{u u u}=A x_{v v v}+a^{\prime \prime} x_{u u}+h^{\prime \prime} x_{u v}+b^{\prime \prime} x_{v v}+l^{\prime \prime} x_{u}+m^{\prime \prime} x_{v}+d^{\prime \prime} x,
\end{aligned}
$$

but no other third-order differential equations.

From (7) we find that

$$
\begin{aligned}
\bar{x}_{u}= & (a+\alpha) x_{u u}+(h+\beta) x_{u v}+b x_{v v}+\left(l+\alpha_{u}+\gamma\right) x_{u}+\left(m+\beta_{u}\right) x_{v} \\
& +\left(d+\gamma_{u}\right) x \\
\bar{x}_{v}= & a^{\prime} x_{u u}+\left(h^{\prime}+\alpha\right) x_{u v}+\left(\beta+b^{\prime}\right) x_{v v}+\left(l^{\prime}+\alpha_{v}\right) x_{u}+\left(m^{\prime}+\beta_{v}+\gamma\right) x_{v} \\
& +\left(d^{\prime}+\gamma_{v}\right) x .
\end{aligned}
$$

It follows from (17) and (16) that the points $\bar{x}_{u u}, \bar{x}_{u v}, \bar{x}_{v v}$ lie in $S(2,0)$ if, and only if,

$$
\beta+b^{\prime}=0, \quad b=0, \quad A(a+\alpha)=0 .
$$

* V. G. Grove, The transformation $C$ of nets in hyperspace, these Transactions, vol. 33 (1931), pp. 733-741. 
If we use (18) we may write equations (17) in the form

$$
\begin{aligned}
\bar{x}_{u}= & (a+\alpha) x_{u u}+\left[\left(l+\alpha_{u}+\gamma\right)-\alpha\left(h-b^{\prime}\right)\right] x_{u}+\left[m-b_{u}^{\prime}+b^{\prime}\left(h-b^{\prime}\right)\right] x_{v} \\
& +\left[d+\gamma_{u}-\gamma\left(h-b^{\prime}\right)\right] x+\left(h-b^{\prime}\right) \bar{x}, \\
\bar{x}_{v}= & a^{\prime} x_{u u}+\left[l^{\prime}+\alpha_{v}-\alpha\left(h^{\prime}+\alpha\right)\right] x_{u}+\left[m^{\prime}-b_{v}^{\prime}+\gamma+b^{\prime}\left(h^{\prime}+\alpha\right)\right] x_{v} \\
& +\left[d^{\prime}+\gamma_{v}-\gamma\left(h^{\prime}+\alpha\right)\right] x+\left(h^{\prime}+\alpha\right) \bar{x} .
\end{aligned}
$$

Some of the integrability conditions of system (16) with $b=0$ are

$$
\begin{gathered}
A a^{\prime}=0, a^{2}+a^{\prime} h+a_{v}=a^{\prime} a^{\prime \prime}+a h^{\prime}+a^{\prime} b^{\prime}+a_{u}^{\prime}+l^{\prime}, \\
b^{\prime}\left(h-b^{\prime}\right)+m-b_{u}^{\prime}=a^{\prime} b^{\prime \prime} .
\end{gathered}
$$

A. Suppose first that $A \neq 0, a^{\prime}=0$. Under conditions (18) equations (19) may be written in the form

$$
\begin{aligned}
& \bar{x}_{u}=\left[l-a_{u}+\gamma+a\left(h-b^{\prime}\right)\right] x_{u}+\left[d+\gamma_{u}-\gamma\left(h-b^{\prime}\right)\right] x+\left(h-b^{\prime}\right) \bar{x} \\
& \bar{x}_{v}=\left[m^{\prime}-b_{v}^{\prime}+\gamma+b^{\prime}\left(h^{\prime}-a\right)\right] x_{v}+\left[d^{\prime}+\gamma_{v}-\gamma\left(h^{\prime}-a\right)\right] x+\left(h^{\prime}-a\right) \bar{x} .
\end{aligned}
$$

It follows therefore that if $A \neq 0, a^{\prime}=0$, the surfaces $S$ and $\bar{S}$ sustain $C$ nets, and the lines $g$ joining corresponding points $x$ and $\bar{x}$ form a congruence $G$, the developables of $G$ intersecting these surfaces in their $C$ nets.

B. Suppose that $A=0$. Under this condition another integrability condition of system (16) is $b^{\prime \prime}=0$. Equations (19) may now be written in the form

$$
\begin{aligned}
\bar{x}_{u}= & (a+\alpha) x_{u u}+\left[l+\alpha_{u}+\gamma-\alpha\left(h-b^{\prime}\right)\right] x_{u}+\left[d+\gamma_{u}-\gamma\left(h-b^{\prime}\right)\right] x \\
& +\left(h-b^{\prime}\right) \bar{x}, \\
\bar{x}_{v}= & a^{\prime} x_{u u}+\left[l^{\prime}+\alpha_{v}-\alpha\left(h^{\prime}+\alpha\right)\right] x_{u}+\left[m^{\prime}-b_{v}^{\prime}+\gamma+b^{\prime}\left(h^{\prime}+\alpha\right)\right] x_{v} \\
& +\left[d^{\prime}+\gamma_{v}-\gamma\left(h^{\prime}+\alpha\right)\right] x+\left(h^{\prime}+\alpha\right) \bar{x} .
\end{aligned}
$$

It follows that the tangent to $v=$ const. on $\bar{S}$ intersects the osculating plane to $v=$ const. on $S$. The tangent planes to $S$ and $\bar{S}$ at $x$ and $\bar{x}$ respectively intersect in a point; they will intersect in a line if, and only if, $a^{\prime}=a+\alpha=0$, that is, if, and only if, the parametric nets on $S$ and $\bar{S}$ are in relation $C$. In this latter case the lines joining corresponding points $x$ and $\bar{x}$ form a congruence.

\section{The parabolic case}

Let us consider the parabolic case. If we differentiate $\bar{x}$ defined by (8) with respect to $u$ and $v$, we obtain

$$
\begin{aligned}
& \bar{x}_{u}=x_{u u u}+\alpha x_{u u}+\beta x_{u v}+\left(\alpha_{u}+\gamma\right) x_{u}+\beta_{u} x_{v}+\gamma_{u} x, \\
& \bar{x}_{v}=x_{u u v}+\alpha x_{u v}+\beta x_{v v}+\alpha_{v} x_{u}+\left(\beta_{v}+\gamma\right) x_{v}+\gamma_{v} x .
\end{aligned}
$$

It follows therefore that if the points $\bar{x}_{u}, \bar{x}_{v}$ lie in $S(2,0)$ the functions $x$ must satisfy a system of differential equations of the form 


$$
\begin{aligned}
& x_{u u u}=a x_{u u}+h x_{u v}+b x_{v v}+l x_{u}+m x_{v}+d x, \\
& x_{u u v}=a^{\prime} x_{u u}+h^{\prime} x_{u v}+b^{\prime} x_{v v}+l^{\prime} x_{u}+m^{\prime} x_{v}+d^{\prime} x .
\end{aligned}
$$

It follows therefore that $S(3,0)$ is of dimensions no higher than seven.

Subcase a. Suppose that $S(3,0)$ is of seven dimensions, that is, that the functions $x$ do not satisfy a third third-order differential equation.

The system (24) has the following integrability conditions*:

$$
\begin{gathered}
b=0, h=b^{\prime}, a_{v}=a_{u}^{\prime}+a^{\prime} h^{\prime}+l^{\prime}, \\
h_{v}+a h^{\prime}+l=h_{u}^{\prime}+a^{\prime} h+h^{\prime 2}+m^{\prime}, \\
a b^{\prime}+m=b_{u}^{\prime}+b^{\prime} h^{\prime}, l_{v}+a l^{\prime}=l_{u}^{\prime}+a^{\prime} l+h^{\prime} l^{\prime}+d^{\prime}, \\
m_{v}+a m^{\prime}+d=m_{u}^{\prime}+a^{\prime} m+h^{\prime} m^{\prime}, \\
d_{v}+a d^{\prime}=d_{u}^{\prime}+a^{\prime} d+d^{\prime} h^{\prime} .
\end{gathered}
$$

It follows from (23) and (24) that the functions $\bar{x}_{u}$ and $\bar{x}_{v}$ are defined by the expressions

$$
\begin{aligned}
(26) \bar{x}_{u}= & (a+\alpha) x_{u u}+(h+\beta) x_{u v}+\left(l+\alpha_{u}+\gamma\right) x_{u}+\left(m+\beta_{u}\right) x_{v}+\left(d+\gamma_{u}\right) x, \\
\bar{x}_{v}= & a^{\prime} x_{u u}+\left(h^{\prime}+\alpha\right) x_{u v}+\left(b^{\prime}+\beta\right) x_{v v}+\left(l^{\prime}+\alpha_{v}\right) x_{u}+\left(m^{\prime}+\beta_{v}+\gamma\right) x_{v} \\
& +\left(d^{\prime}+\gamma_{v}\right) x .
\end{aligned}
$$

From (26) we find that the points $\bar{x}_{u u}, \bar{x}_{u v}, \bar{x}_{v v}$ lie in the space $S(2,0)$ if and only if

$$
\alpha+h^{\prime}=0, \beta+b^{\prime}=0 .
$$

Therefore the surface $\bar{S}$ generated by the point $\bar{x}$ defined by the expression

$$
\bar{x}=x_{u u}-h^{\prime} x_{u}-b^{\prime} x_{v}+\gamma x
$$

is such that the two-osculating space $\bar{S}(2,0)$ at $\bar{x}$ coincides with the space $S(2,0)$ at $x$ for every choice of $\gamma$.

If we make use of equation (28) we may write equation (26) in the form

$$
\begin{aligned}
\bar{x}_{u} & =\mu x_{u}+f x+A \bar{x}, \\
\bar{x}_{v} & =r x_{u}+\mu x_{v}+g x+B \bar{x},
\end{aligned}
$$

wherein

$$
\begin{aligned}
& \mu=h^{\prime}\left(a-h^{\prime}\right)+l-h_{u}^{\prime}+\gamma=a^{\prime} b^{\prime}+m^{\prime}-b_{v}^{\prime}+\gamma, \\
& f=d+\gamma_{u}-\gamma\left(a-h^{\prime}\right), \quad A=a-h^{\prime}, \quad B=a^{\prime}, \\
& g=d^{\prime}+\gamma_{v}-a^{\prime} \gamma, \quad r=a^{\prime} h^{\prime}+l^{\prime}-h_{v}^{\prime} .
\end{aligned}
$$

* Lane, Surfaces, p. 792. 
We may readily verify that as $x(\bar{x})$ moves along the curve $v=$ const. on $S(\bar{S})$ the point

$$
y=\bar{x}-\mu x, \quad r \neq 0,
$$

describes a curve whose tangent at $y$ is the line $g$ joining $x$ to $\bar{x}$. Moreover there exists no other curve on $S(\bar{S})$ along which $x(\bar{x})$ may move so that the line $g$ will generate a developable surface. We may readily verify that the lines $g$ generate a congruence $G$ composed of the tangents to a one-parameter family of asymptotic curves on the surface generated by the point $y$. However the point $y$ defined by the expression

$$
y=\bar{x}-\mu x, \quad r=0,
$$

is a fixed point, and the lines $g$ form a bundle of lines through this fixed point.

Subcase b. Suppose that the space $S(3,0)$ is of six dimensions.

A. The points $x_{u v}, x_{v v}$, as may be seen from (23), will lie in the space $S(2,0)$ if

$$
\beta=-h, \quad \alpha=-h^{\prime},
$$

and if $x$ satisfies the equations (24) and a differential equation of the form

$$
x_{v v v}=a^{\prime \prime} x_{u u}+h^{\prime \prime} x_{u v}+b^{\prime \prime} x_{v v}+l^{\prime \prime} x_{u}+m^{\prime \prime} x_{v}+d^{\prime \prime} x .
$$

Some of the integrability conditions of the system composed of equations (24) and (32) are

$$
b=0, h=b^{\prime}, m+b^{\prime}\left(a-h^{\prime}\right)-b_{u}^{\prime}=0 .
$$

We may readily verify that the point $\bar{x}$ defined by

$$
\bar{x}=x_{u u}-h^{\prime} x_{u}-b^{\prime} x_{v}+\gamma x
$$

generates a surface $\bar{S}$ whose two-osculating space $\bar{S}(2,0)$ at $\bar{x}$ coincides with the two-osculating space $S(2,0)$ of $S$ at $x$. Moreover the tangent planes to $S$ at $x$ and $\bar{S}$ at $\bar{x}$ intersect in a line $h$. The projectivity determined on $h$ by the pencils of tangent lines to $S$ and $\bar{S}$ at $x$ and $\bar{x}$ is parabolic. The lines $g$ joining $x$ to $\bar{x}$ form a congruence of tangents to a one-parameter family of asymptotic curves on a surface.

B. The space $\bar{S}(2,0)$ of $\bar{S}$ at $\bar{x}$ will also coincide with the space $S(2,0)$ at $x$ if

$$
\beta+b^{\prime}=0,
$$

and if $x$ satisfies equations (24) and a differential equation of the form 


$$
x_{u u v}=a^{\prime \prime} x_{u u}+h^{\prime \prime} x_{u v}+b^{\prime \prime} x_{v v}+l^{\prime \prime} x_{u}+m^{\prime \prime} x_{v}+d^{\prime \prime} x .
$$

Two of the integrability conditions of such a system are

$$
b=0, \quad b^{\prime}=0 \text {. }
$$

It follows therefore that any point defined by the expression

$$
\bar{x}=x_{u u}+\alpha x_{u}+\gamma x
$$

( $\alpha$ and $\gamma$ arbitrary) in the osculating plane to $v=$ const. on $S$ at $x$ generates $a$ surface $\bar{S}$ whose two-osculating space $\bar{S}(2,0)$ at $\bar{x}$ coincides with the space $S(2,0)$ at $x$. The tangent planes to $S$ and $\bar{S}$ at $x$ and $\bar{x}$ intersect in $a$ point.

Suppose that in the expression (4) $A=B=C=0$. By a transformation of the curvilinear coordinates we may write (4) in the form

$$
\bar{x}=x_{u}+\gamma x .
$$

By repeated differentiations we find that $\bar{S}(2,0)$ coincides with $S(2,0)$ if, and only if, the functions $x$ satisfy a system of differential equations composed of equations of the form (24) and (34). It follows that the space $S(3,0)$ of $S$ at $x$ is of six dimensions. Conversely if the functions satisfy such a system, a point $\bar{x}$ defined by (35) generates a surface of the required type.

\section{The conjugate case}

Suppose now that $S$ sustains a conjugate net. By proper choice of the parameters we may take this net to be the parametric net. The functions $x$ therefore satisfy an equation of the Laplace type

$$
x_{u v}=a x_{u}+b x_{v}+c x .
$$

It follows from (36) that $S(2,0)$ is a space of four dimensions and that $S(3,0)$ is a space of not more than six dimensions.

Let the point $\bar{x}$ be defined by the expression

$$
\bar{x}=A x_{u u}+C x_{v v}+\alpha x_{u}+\beta x_{v}+\gamma x,
$$

wherein not both $A$ and $C$ are zero.

A. Suppose first that $\bar{S}(3,0)$ is of six dimensions. We find readily that there exist no surfaces $\bar{S}$ distinct from $S$ such that the spaces $\bar{S}(2,0)$ and $S(2,0)$ coincide.

B. Suppose that $S(3,0)$ is of five dimensions. We find from (37) that

$$
\begin{aligned}
\bar{x}_{u}= & A x_{u u u}+(A+\alpha) x_{u u}+\left(b C+C_{u}\right) x_{v v}+\left[C\left(a_{v}+a^{2}\right)+a \beta+\alpha_{u}+\gamma\right] x_{u} \\
& +\left[C\left(c+a b+b_{v}\right)+b \beta+\beta_{u}\right] x_{v}+\left[C\left(c_{v}+a c\right)+\beta c+\gamma_{u}\right] x .
\end{aligned}
$$

A symmetrical expression obtains for $\bar{x}_{v}$. It follows that if $A \neq 0$, the func- 
tions $x$ satisfy an equation of the form

$$
x_{u u u}=a^{\prime} x_{u u}+b^{\prime} x_{v v}+l^{\prime} x_{u}+m^{\prime} x_{v}+d^{\prime} x .
$$

In order that $\bar{x}_{v}$ lie in the space $S(2,0)$, and that $S(3,0)$ be a space of five dimensions the coefficient $C$ must be zero.

Some of the integrability conditions of the system composed of equations (36) and (39) are

$$
b^{\prime}=0, \quad m^{\prime}=0, \quad a_{v}^{\prime}-a_{u}=c+a b+a_{u} .
$$

Hence the curves $v=$ const. on $S$ are plane curves. With the expression for $\bar{x}_{v}$ and $C=0$, we find that $\bar{x}_{v v}$ lies in $S(2,0)$ if, and only if, $\beta=0$. Hence $\bar{x}$ lies in the plane of the curve $v=$ const.

If we set $A=1$, we find that the points $\bar{x}_{u}, \bar{x}_{v}$ are defined by the expressions

$$
\begin{aligned}
\bar{x}_{u}= & {\left[l^{\prime}+\gamma+\alpha_{u}-\alpha\left(a^{\prime}+\alpha\right)\right] x_{u} } \\
& +\left[d^{\prime}+\gamma_{u}-\gamma\left(a^{\prime}+\alpha\right)\right] x+\left(a^{\prime}+\alpha\right) \bar{x}, \\
\bar{x}_{v}= & \left(c+a b+a_{u}+\alpha_{v}\right) x_{u}+\left(b^{2}+b_{u}+\alpha b+\gamma\right) x_{v} \\
& +\left(c_{u}+b c+\alpha c+\gamma_{v}-a \gamma\right) x+a \bar{x} .
\end{aligned}
$$

The tangent planes to $S$ and $\bar{S}$ at $x$ and $\bar{x}$ intersect in a line. Hence if $S(3,0)$ is a space of five dimensions, and if $S$ sustains a conjugate net, the point $\bar{x}$ defined by (37) will describe a surface $\bar{S}$ whose two-osculating space $S(2,0)$ at $\bar{x}$ coincides with $S(2,0)$ at $x$ if and only if each curve of one of the component families of curves of the conjugate net is a plane curve, and the point $\bar{x}$ is a point in the plane of the curve. The lines $g$ joining $x$ and $\bar{x}$ form a congruence.

Suppose that $\bar{x}$ lies in the tangent plane of $S$ at $x$, that is, suppose that in (37) $A=C=0$. We readily verify that if $S(3,0)$ is of six dimensions the space $\bar{S}(2,0)$ at $\bar{x}$ cannot coincide with the space $S(2,0)$ at $x$ for distinct surfaces $S$ and $\bar{S}$. If $S(3,0)$ is a space of five dimensions, the point $\bar{x}$ must lie in the tangent to one of the curves of the conjugate net, and that family of curves is a family of plane curves.

\section{The ASYMptotic CASE}

Suppose that $S$ sustains a one-parameter family of asymptotic curves. Let the notation be so chosen that the curves $v=$ const. are the asymptotics. It follows that the functions $x$ defining $S$ satisfy the differential equation

$$
x_{u u}=a x_{u}+b x_{v}+c x .
$$

It follows that the space $S(3,0)$ is a space of six dimensions at most. 
Let $\bar{x}$ be defined by an expression of the form

$$
\bar{x}=B x_{u v}+C x_{v v}+\alpha x_{u}+\beta x_{v}+\gamma x,
$$

wherein not both $B$ and $C$ are zero.

A. We may readily verify that if $S(3,0)$ is a space of six dimensions, there exists no surface $\bar{S}$ distinct from $S$ with the desired property.

B. Suppose therefore that $S(3,0)$ is a space of five dimensions. It follows from (42) that the points $\bar{x}_{u}$ and $\bar{x}_{v}$ are in $S(2,0)$ if, and only if, $C=0$, and the functions $x$ satisfy a differential equation of the form

$$
x_{u v v}=h^{\prime} x_{u v}+b^{\prime} x_{v v}+l^{\prime} x_{u}+m^{\prime} x_{v}+d^{\prime} x .
$$

Two of the integrability conditions of the system composed of equations (41) and (43) are

$$
b=0, \quad c-b_{u}^{\prime}+b^{\prime}\left(a-b^{\prime}\right)=0 .
$$

It follows therefore that the surface $S$ is ruled.

If in (42) we set $C=0, B=1$, we find that

$$
\begin{aligned}
\bar{x}_{u}= & (a+\beta) x_{u v}+\left(a_{v}+a \alpha+\alpha_{u}+\gamma\right) x_{u} \\
& +\left(c+\beta_{u}\right) x_{v}+\left(c_{v}+\alpha c+\gamma_{u}\right) x, \\
\bar{x}_{v}= & \left(a^{\prime}+\alpha\right) x_{u v}+\left(\beta+b^{\prime}\right) x_{v v}+\left(l^{\prime}+\alpha_{v}\right) x_{u} \\
& +\left(m^{\prime}+\beta_{v}+\gamma\right) x_{v}+\left(d^{\prime}+\gamma_{v}\right) x .
\end{aligned}
$$

The points $\bar{x}_{u u}, \bar{x}_{u v}, \bar{x}_{v v}$ lie in $S(2,0)$ if, and only if, $\beta=-b^{\prime}$. Equation (45) may be written in the form

$$
\begin{aligned}
\bar{x}_{u}= & {\left[a_{v}+a \alpha+\alpha_{u}+\gamma-\alpha\left(a-b^{\prime}\right)\right] x_{u}+\left[c_{v}+\alpha c+\gamma_{u}-\gamma\left(a-b^{\prime}\right)\right] x } \\
& +\left(a-b^{\prime}\right) \bar{x}, \\
\bar{x}_{v}= & {\left[l^{\prime}+\alpha_{v}-\alpha\left(a^{\prime}+\alpha\right)\right] x_{u}+\left[m^{\prime}-b_{v}^{\prime}+\gamma+b^{\prime}\left(a^{\prime}+\alpha\right)\right] x_{v} } \\
& +\left[d^{\prime}+\gamma_{v}-\gamma\left(a^{\prime}+\alpha\right)\right] x+\left(a^{\prime}+\alpha\right) \bar{x} .
\end{aligned}
$$

The point $\bar{x}$ defined by the expression

$$
\bar{x}=x_{u v}+\alpha x_{u}-b^{\prime} x_{v}+\gamma x
$$

for arbitrary values of $\alpha$ and $\gamma$ generates a surface $\bar{S}$ whose two-osculating space $\bar{S}(2,0)$ at $\bar{x}$ coincides with the two-osculating space $S(2,0)$ of $S$ at $x$.

The point $r$ defined by the expression $r=x_{u}-b^{\prime} x$ is readily characterized as the only point, on the generator through $x$ of the ruled surface, describing a surface for which the osculating plane to the curve $u=$ const. at $r$ lies in the space of three dimensions tangent to the ruled surface along the generator through $x$. We find that 


$$
r_{v}+\alpha r=\bar{x}-\left(\alpha b^{\prime}+\gamma+b_{v}^{\prime}\right) x .
$$

It follows that the lines $g$ joining $x$ to $\bar{x}$ form a congruence. The line $g$ passes through $x$ and intersects the tangent line to the curve $u=$ const. on the surface generated by the point $r$.

Suppose that $\bar{x}$ lies in the tangent plane to $S$ at $x$. We readily verify that $\bar{S}(2,0)$ at $\bar{x}$ will coincide with $S(2,0)$ at $x$ if and only if $\bar{x}$ lies in the tangent line of the asymptotic curve on $S$ through $x$, and if the functions $x$ defining the surface satisfy a differential equation of the form (43).

Michigan State College,

East Lansing, Mich. 